\title{
Chikungunya- A Diabetogenic Stress.
}

\author{
Dr. M. Ramakrishnan \\ Kerala state health service department south India
}

\begin{abstract}
Diabetogenic potential of chikungunya fever was suspected during routine management of cases attending my hospital during the 2009 chikungunya epidemic in Kerala, south India. The suspicion was given due attention because doctors were treating chikungunya with steroids like methyl prednisolone, a known diabetogenic drug and many patients had one ... or more components of metabolic syndrome..I made an attempt to evaluate the interaction between chikungunya and diabetes using the patient and laboratory data that was naturally available to me till cases ceased to report.
\end{abstract}

Key Words: Chikungunya 2.Diabetogenesis.

\section{Indroduction}

Chikungunya is an insect-born virus of the genus alpha virus, family togaviridae that is transmitted to human by virus-carrying mosquito aedes aegypti.Recent epidemic in different parts of the world including kerala has been due to aedes albopictus mosquito. The change was due to a mutation in the virus. Symptoms of the disease include a fever upto 40 degree $\mathrm{C}$, petechial or maculo-papular rash of the trunk, face or limbs and sudden onset of symmetrical polyarthralgia or arthritis. There will be nonspecific symptoms like head ache, photophobia etc. The acute phase is treated with paracetamol and NSAID. Chloroquine is also advised by virtue of its anti inflammatory action ,but doctors preferred and used steroid for definite results. Recovery from chikungunya varies with age. Younger patients recover within 5-15 days .Arthralgia may persist for weeks to months irrespective of treatment modality followed.

I noticed that recovery from acute phase of chikungunya was slow in diabetics , and their blood sugar used to go up quite early during the disease. Also I noted that severity and chronicity of the disease In prediabetics and diabetics were reduced when measures to addres s dysglycemia were followed.

\section{Material and method}

It was the mansoon of 2009 , and beside chikungunya ,there were other fevers like viral fever, hepatitis,enteric fever and respiratory tract infection. Often more than one type of fever were present in different members of same family making the diagnosis difficult. Chikungunya cases were diagnosed based on the clinical features and virological diagnosis of random cases using government facilities.

I examined more than one thousand cases of fever during that period out of which 420 were chikungunya cases. My patients included cases partly treated by other doctors also among which most of the patients were on steroids from day one itself. All routine tests including blood sugar estimation and platelet count were done to pick up serious cases like dengue fever and pneumonia. Along with this, routine screening for diabetes continued as ever, and I included a history of chikungunya also as a criteria for screening for obvious reasons. More over any disease precipitated or aggravated along with or after chikungunya were also carefully watched and their relationship to dysglycemia noted. The effect of steroid therapy initiated by other doctors were noted while trying to taper it as early as possible.

\section{Observations.}

1. Detection rate of diabetes during opportunistic screening increased by 20 percent when recent history of chikungunya was also taken as a criteria for screening of adults for diabetes.

2. Different modalities of increase in medicine requirement were noted in few diabetics following chikungunya as follows suggesting an existing diabetogenic stress in operation.

\begin{tabular}{|c|c|c|c|c|}
\hline Sl No & INITIAL GROUP & CHANGED TO & $\begin{array}{l}\text { APPROXIMATE } \\
\text { PERCENTAGE }\end{array}$ & $\mathrm{P}-$ Value \\
\hline 1 & $\begin{array}{ll}\text { Diet } & \text { alone } \\
10 & \end{array}$ & One O.H.A 2 & 20 & 0.2 \\
\hline 2 & O.H.A & O.H.A & 40 & 0.38 \\
\hline 3 & $\begin{array}{lll}\text { Low } & \text { dose } & \text { O.H.A } \\
10 & & \\
\end{array}$ & $\begin{array}{lll}\text { High } & \text { dose } & \text { O.H.A } \\
\end{array}$ & 20 & 0.2 \\
\hline
\end{tabular}


Chikungunya- A Diabetogenic Stress

\begin{tabular}{|l|lr|l|l|l|l|}
\hline 4 & $\begin{array}{l}\text { Multiple } \\
16\end{array}$ & O.H.A & $\begin{array}{l}\text { Oral medicine + Short course } \\
\text { insulin } \\
5\end{array}$ & 30 & 0.31 \\
\hline 5 & $\begin{array}{l}\text { Short course insulin } \\
5\end{array}$ & $\begin{array}{l}\text { Chronic insulin dependency } \\
3\end{array}$ & 60 & 0.5 \\
\hline
\end{tabular}

All observations are statistically significant

3. Even in mild diabetics, complications like painful neuritis and frozen shoulder were precipitated in few person following chikungunya.

4. Chronic arthralgia of chikungunya showed good response to low glycemic index diet.

5.Dysglycemia mediated alterations in immune status were noted following chikungunya .In this group I came across cases like flare up of Myasthenia gravis and tuberculosis both of which showed good response to management of the dysglycemia alone. I postulate this as clinical criteria for viral diabetogenesis in human and name as RAMAKRISHNAN PHENOMENON.

\section{Discussion.}

The incidence and prevalence of diabetes are increasing world wide making it the biggest non communicable public health problem. Along with this the number of undiagnosed diabetes is also on the increase despite availability of definite screening and treating methods. One thing that contribute to abundance of undiagnosed diabetes and diabetes fluctuations are newer and newer diabetogenic stresses like infections, drugs and other environmental factors either alone or in combination during different occasions in an individuals lifetime.

Chikungunya is widely distributed in Africa ,middle east and Asia,affecting millions of people every year. Attention is sought about the diabetogenic potential of chikungunya epidemic ,and the need to translate appropriate preventive measures. It will be evident that steroids have to be used with caution in this disease.

Experiments in mice regarding viral diabetogenesis report that it is the changes induced in lymphocytes and not the virus directly leading to diabetogenesis. Similar mechanism may be operating in viral diabetogenesis in human as well.

\section{Conclusions.}

1. Chikungunya is a diabetogenic stress and set an example for viral diabetogenesis in human.

2. The stress is powerful enough to revise medical treatment in significant percentage of diabetics.

3. Every chikungunya case should be screened for diabetes sooner or later.

4. Chikungunya cases are susceptible for drug induced diabetes even with a short course of steroid.

5. The diabetogenic potential of the virus may be an effect of the mutation that helped the virus to inhabit aedes albopictus mosquito, the vector of recent epidemics.

6. Multiple diabetogenic stresses contribute to abundance of undiagnosed diabetics and should be a topic of concern in preventive diabetology..

I express my respect and gratitude towards

\section{Acknowledgement.}

1.Dr.K.P.Ramamoorthy M.D; Ph.D,

Emeritus Professor of Medicine .Government Medical College Calicut and

Research Guide, University of Calicut for supervising this study.

2.Dr.V.Mohan M.D :F.R.C.P; Ph.D ;D.Sc ;F.N.A.Sc

Scientist, Chennai, for examining this paper

\section{Further reading}

[1] Effect of environmental factors on the development of IDDM Yoon JW, Kim CJ, Pak CY, Mc Arthur RG. Department of microbiology and Infectious Diseases. Julia McFarlane Diabetes Research Unit University of Calgury ,Alberta, Canada.

[2] Possible mechanism in the pathogenesis of virus induced diabetes by the same workers above.

[3] Chikungunya;Wikipedia.

[4] Chikungunya in India; Information sheet W.H.O;S.E.A.R.O ,New Delhi.

[5] A study on Chikungunya outbreak during during 2007 in Kerala, South India; Kannan et al,I.C.M.R. Madurai,India. Indian journal of Med Res.129, March2009. Pp311 to 315.

[6] Diabetes and myasthenia gravis- Article in internet by Dr.Robert. M. Cohen M.D, Associate professor of medicine Univercity of Cincinati.

[7] Myasthenia gravis and Diabetes mellitus.Internet- Researchers ; Toho University .Tokyo

[8] Diabetes mellitus and Frozen shoulder or Capsular fibroplasias-The mystery unfolding.Int.J. Diab. Devoloping Countries (1999) Vol.19.

[9] A.G. Archer et al. The natural history of acute painful neuropathy in diabetes mellitus. Topic Abstract; Internet. 